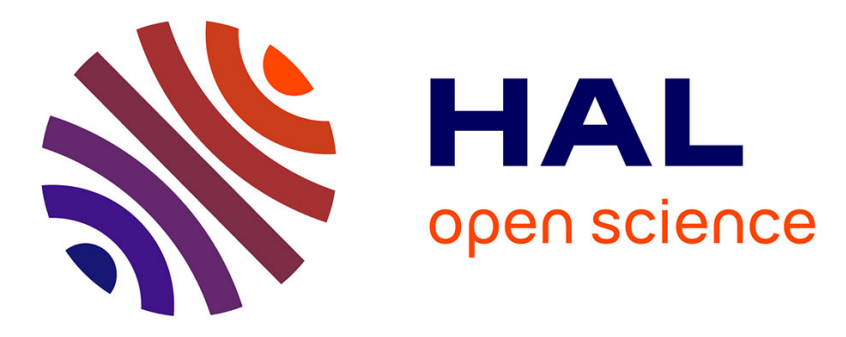

\title{
Travail ou santé? Le dilemme des saisonniers agricoles migrants
}

\author{
Frédéric Décosse
}

\section{To cite this version:}

Frédéric Décosse. Travail ou santé? Le dilemme des saisonniers agricoles migrants. Thébaud-Mony A., Davezies P, Vogel L. \& Volkoff S. Les risques du travail. Pour ne pas perdre sa vie à la gagner, La Découverte, 2015. halshs-01135695

\section{HAL Id: halshs-01135695 https://shs.hal.science/halshs-01135695}

Submitted on 25 Mar 2015

HAL is a multi-disciplinary open access archive for the deposit and dissemination of scientific research documents, whether they are published or not. The documents may come from teaching and research institutions in France or abroad, or from public or private research centers.
L'archive ouverte pluridisciplinaire HAL, est destinée au dépôt et à la diffusion de documents scientifiques de niveau recherche, publiés ou non, émanant des établissements d'enseignement et de recherche français ou étrangers, des laboratoires publics ou privés. 
DECOSSE, Frédéric - 2015, « Travail ou santé ? Le dilemme des saisonniers agricoles migrants ». In ThébaudMony A., Davezies P, Vogel L. \& Volkoff S. (dir), Les risques du travail. Pour ne pas perdre sa vie à la gagner. Paris : La Découverte.

\title{
Travail ou santé ? Le dilemme des saisonniers agricoles migrants
}

\author{
Frédéric Décosse, Aix Marseille Université, CNRS, LEST UMR 7317
}

Comment sont produits les fruits et légumes vendus à longueur d'année en supermarché ? Derrière le «manger sain » promu par les autorités de santé, quelles conditions de travail et quels accès à la santé pour les travailleurs de la terre, notamment pour le précariat migrant qu'emploie l'agriculture industrielle? L'arboriculture et le maraîchage intensifs exposent les ouvriers agricoles tant aux risques professionnels traditionnels du secteur qu'à des risques de type industriel. Selon l'enquête «Surveillance médicale des expositions aux risques professionnels » (SUMER), ces salariés travaillent fréquemment plus de quarante heures par semaine, d'où des astreintes la nuit, le dimanche et les jours fériés, et des temps de repos limités et fragmentés. Les contraintes physiques y ont globalement augmenté depuis vingt ans : les salariés sont soumis à des postures de travail inconfortables (station debout prolongée avec piétinement, travail accroupi, à genoux ou en torsion) et à des contraintes articulaires associées au port de charges lourdes et à des gestes répétitifs (en viticulture par exemple, la taille impose aux ouvriers une moyenne quotidienne de 20000 coups de sécateur); ils sont également exposés aux intempéries, aux machines vibrantes et au bruit. L'enquête précitée souligne l'exposition de ces salariés à des poussières organiques et végétales, ainsi qu'à des produits chimiques (pour $53 \%$ d'entre eux) et autres substances cancérogènes (poussières d'engrais, huiles, gasoil et fumées de carburants, formaldéhyde, etc.). Selon les médecins du travail, la prévention des risques physiques et chimiques est insuffisante dans $35 \%$ à $45 \%$ des cas et l'organisation du travail est jugée mauvaise dans $16 \%$ des situations observées. 
La Mutualité sociale agricole (MSA) note que le taux de fréquence des accidents de travail oscille entre quarante et cinquante par million d'heures travaillées, soit environ dix de plus que la moyenne du secteur agricole et vingt de plus que dans le régime général. Le taux d'incapacité permanente partielle moyen est de $10 \%$. Les salariés extracommunautaires sont davantage concernés par les accidents graves (18\%) que leurs collègues français et autres ressortissants de l'union européenne (8\%). L'arboriculture et le maraîchage concentrent $10 \%$ des accidents mortels. Ce sont donc des domaines d'activité professionnelle particulièrement accidentogènes, où la gravité des accidents est forte en comparaison avec d'autres secteurs d'emploi réputés dangereux comme le BTP. Les accidents de travail déclarés sont principalement liés à des pertes d'équilibre, des opérations de manutention et à l'utilisation de « matériels et accessoires ». Ils occasionnent majoritairement des plaies au niveau des mains et de la tête, des douleurs à l'effort au niveau du rachis, des entorses au niveau des membres inférieurs et des fractures au niveau des pieds. Le secteur concentre également une bonne part des quelque 3600 maladies professionnelles reconnues en agriculture - des troubles musculo-squelettiques pour la plupart.

\section{Travailleurs migrants et délocalisation sur place}

En raison de ces conditions de travail dégradées et dangereuses, mais aussi et surtout de la faiblesse des salaires, le personnel des vergers et des serres de l'agriculture intensive du Sud de la France est presque exclusivement constitué de migrants originaires du Maghreb, d'Europe de l'Est et plus récemment d'Amérique du Sud : étrangers en situation de séjour plus ou moins précaire, sans-papiers, étudiants, salariés d'entreprises de travail temporaire étrangères ou encore saisonniers recrutés par le biais de l'Office des migrations internationales (OMI, devenu aujourd'hui OFII). La présence temporaire sur le territoire de cette dernière catégorie de travailleurs migrants pose des problèmes spécifiques en termes de santé et sécurité au travail. Faute de moyens humains supplémentaires dans les services de médecine du travail durant la saison, les « OMI » sont peu concernés par l'examen d'embauche, ce qui les 
prive à priori d'un suivi médical systématique et contribue directement à l'effacement des traces de leur exposition professionnelle aux toxiques et autres agents pathogènes. Leurs employeurs jugeant inutile d'investir dans leur formation, les saisonniers manipulent des outils et produits dangereux sans préparation adéquate, ni déclaration auprès des services de santé au travail, ni surveillance médicale renforcée. Leur manque d'insertion au sein du collectif de travail et leur isolement vis-à-vis des syndicats les empêchent de développer des stratégies collectives de protection de leur santé. Enfin, l'obligation qui leur est faite de rentrer dans leur pays d'origine et le refus (illégal) de la Mutualité sociale agricole de maintenir leurs droits sociaux à la fin de la saison les éloignent de toute possibilité de se soigner et se faire reconnaître une pathologie professionnelle.

Qui sont les saisonniers OMI? Comment se construit l'invisibilisation de leurs atteintes et l'externalisation de leurs pathologies professionnelles vers leur pays d'origine? Venus du Maroc et de Tunisie, ils sont quelques milliers à être annuellement employés par des maraîchers et arboriculteurs du Sud de la France pour une période allant de quatre à six mois. Plus que le caractère discontinu de leur emploi sur le territoire national, c'est la précarité de leur permis de résidence qui en fait une population laborieuse vulnérable. Leur droit au séjour est en effet strictement lié à leur contrat de travail et donc à leur employeur qui décide souverainement s'il le renouvelle ou non l'année suivante. Ce dispositif d'immigration placée et jetable confère au patronat le moyen de discipliner la force de travail et de l'amener à mettre sa santé entre parenthèses, d'autant que, compte tenu de leur rareté, les contrats saisonniers OMI s'achètent environ 6000 euros sous le manteau et les migrants doivent donc s'endetter pour obtenir le droit de travailler en France. Dans un tel contexte, l'ineffectivité de leur droit à la santé se construit au carrefour des pratiques de déni des employeurs (refus de déclaration ou négation du caractère professionnel des accidents de travail, injonction à la reprise anticipée du travail) et de la Mutualité sociale agricole (consolidation hâtive des accidents de travail, suspension des indemnités journalières au terme du contrat de travail, etc.) et des pratiques de nonrecours des salariés eux-mêmes (sous-déclaration des accidents de travail). 


\section{Entre externalisation et résistances}

Un exemple parmi d'autres : pendant près de vingt ans, M. Hasni a travaillé dans une exploitation maraîchère de Berre-l'Étang. Compte tenu de son ancienneté, l'employeur lui confiait l'encadrement des autres saisonniers OMI, ainsi que la réalisation de tâches qualifiées, telles que la conduite d'un tracteur vétuste (sans arceau de sécurité) pour laquelle il n'a reçu aucune formation. Août 2004. Alors que M. Hasni effectue un virage en sortie de serre, l'engin se renverse et le salarié décède avant d'avoir pu être dégagé. Si le patron déclare effectivement l'accident de travail, la description qu'il fait de ses circonstances [Codetras, 2005, p. 11] vise à faire établir la faute inexcusable de l'ouvrier, susceptible de réduire ses droits à réparation :

Mon employé effectuait un arrachage de plants de fin de récolte. J'ai dû m'absenter et je lui ai recommandé de ne pas toucher au tracteur. Malgré mon interdiction, il a quand même voulu évacuer les déchets et s'est servi du tracteur pendant mon absence. Je ne m'explique pas cet accident qui s'est produit sur un terrain plat. Toujours est-il que l'ouvrier a été écrasé sous le tracteur. Il s'agit bien d'un accident survenu pendant le temps de travail, travail qu'il n'aurait pas dû faire.

Mandatés par ses proches, la CFDT et le Collectif de défense des travailleurs agricoles saisonniers (Codetras) sont finalement parvenus à faire reconnaître le caractère plein et entier de l'accident de travail et le capital décès et l'allocation veuvage ont pu être versés à la famille au Maroc. Un grain de sable dans les rouages bien huilés de la division internationale des risques du travail.

\section{Références bibliographiques}

Codetras (2005), Les Omis. Livre noir de l'exploitation des travailleurs étrangers dans l'agriculture des Bouches-du-Rhône. Consultable en ligne sur $<$ http://www.codetras.org $>$.

DECOSSE Frédéric (2011), Migrations sous contrôle. Agriculture intensive et saisonniers marocains sous contrat «OMI», Éditions de l'EHESS, Paris.

Enquête SUMER. Consultable en ligne sur <http://travail-emploi.gouv.fr〉 . 